\title{
Causal Effect Analysis of Demographic Concordance of Physician Trust and Respect in an Emergency Care Setting
}

\author{
Amy $\mathrm{F} \mathrm{Ho}{ }^{1, *}$ \\ Yuan Zhou ${ }^{2, *}$ \\ Jessica J Kirby' \\ Md Mamunur Rahman ${ }^{2}$ \\ Kathryn Tessitore' \\ Yousef Abdel-Raziq' \\ James P d'Etienne' \\ Chet D Schrader' \\ Hao Wang (D) $^{\prime}$

\begin{abstract}
'Department of Emergency Medicine, Integrative Emergency Services, John Peter Smith Health Network, Fort Worth, TX, 76104, USA; ${ }^{2}$ Department of Industrial, Manufacturing, and Systems Engineering, The University of Texas at Arlington, Arlington, TX, 76019, USA

*These authors contributed equally to
\end{abstract} \\ this work
}

Objective: Patient perceptions of physician trust and respect are important factors for patient satisfaction evaluations. However, perceptions are subjective by nature and can be affected by patient and physician demographic characteristics. We aim to determine the causal effect on patient-physician demographic concordance and patient perceptions of physician trust and respect in an emergency care setting.

Methods: We performed a causal effect analysis in an observational study setting. A nearreal-time patient satisfaction survey was sent via telephone to patients within $72 \mathrm{~h}$ of discharge from an emergency department (ED). Patient-trust-physician (PTP) and physicianshow-respect (PSR) scores were measured. Patient and physician demographics (age, gender, race, and ethnicity) were matched. Causal effect was analyzed to determine the direct effect of patient-physician demographic concordance on PTP/PSR scores.

Results: We enrolled 1815 patients. The treatment effect of patient-physician age concordance on PTP scores was $-0.119(\mathrm{p}=0.036)$. Other treatment effect of patientphysician demographic concordance on patient perception of physician trust and respect ranged from -0.02 to $-0.2(\mathrm{p}>0.05)$.

Conclusion: Patient-physician age concordance may cause a negative effect on patient perception of physician trust. Otherwise, patient-physician demographic concordance has no effect on patient perceptions of physician trust and respect.

Keywords: patient, physician, demographics, trust, respect

\section{Introduction}

Patient satisfaction is measured by after-care satisfaction surveys and has been used as a core metric to determine better quality patient-centered care. ${ }^{1,2}$ Further examining patient satisfaction scores linked to these subjective questions revealed that they could be affected by both patient and provider demographics. ${ }^{3,4}$ Results from McFarland et al showed that younger, white patients were predictive of a favorable health-care rating, while non-English speaking patients were predictive of unfavorable health-care satisfaction scores. ${ }^{5,6}$ A study performed by Ommen et al found that patients' age affected the trust to their physicians independently. ${ }^{7}$ Moreover, a national cancer patient experience survey revealed that ethnic patients may report lower satisfaction and less positive experiences in their overall care, similar to the McFarland outcomes. ${ }^{8}$ However, findings are quite controversial in other studies. A retrospective study done at ED showed that elderly patients ( $>65$ years old) had higher patient satisfaction than younger patients. ${ }^{9}$ However, such findings could be
Correspondence: Yuan Zhou Department of Industrial, Manufacturing, and Systems Engineering, The University of Texas at Arlington, 70I S. Nedderman Dr., Arlington, TX, 76019, USA Email yuan.zhou@uta.edu

Hao Wang

Integrative Emergency Services, John

Peter Smith Health Network, 1500

S. Main St., Fort Worth, TX, 76104, USA

Tel + I 817-702-8696

Fax + I 817-702-II43

Email hwang@ies.healthcare 
biased without analyzing health-care provider demographics in relation to patient satisfaction.

In recent years, more studies have reported patient and physician demographic concordance affecting patient satisfaction. An HIV study conducted in New York City found that HIV patients rated their quality of healthcare higher if they are racially/ethnically concordant with their providers. ${ }^{10}$ A primary care physician study found that race concordance seemed to be the primary predictor of perceived ethnic similarity, thus associated with higher ratings of provider trust, satisfaction, and intention to adhere to healthcare management. ${ }^{11}$ Unfortunately, these findings were not corroborated in other studies, which showed less association between patient-physician demographic concordance and general health-care satisfaction. ${ }^{12,13}$ However, among all these studies, common association analytical methods such as correlation and regression were used with no direct causal effect reported between patient-physician demographic concordance and patient satisfaction.

Among patient satisfaction survey questions, some questions are asked to determine patient satisfaction with respect to their health-care providers. Patient responses to these questions can be very subjective, particularly on ratings describing trust and respect between physicians and patients. Trust and respect could be highly variable across different demographics. Therefore, it is important to consider crosscultural disparities between patients and health-care providers. Such disparities could further affect patient-physician communications, thereby subsequently affect patientcentered care. ${ }^{14}$ Therefore, in this study, we aim to determine patient-physician demographics affecting patient perceptions of provider trust and respect by using a causal effect methodology, a more accurate association analysis.

\section{Methods}

\section{Study Design and Setting}

This is a secondary data analysis from a quality improvement project. Data were collected prospectively from October 1, 2018, to September 31, 2019, but were analyzed retrospectively. This study was performed in an urban tertiary public funded hospital ED. The study ED has approximately 125,000 visits per year and is a level 1 trauma center, a comprehensive stroke center, and a chest pain center. Due to secondary data analysis from a quality improvement project, approval for this study was waived by the local Institutional Review Board.

\section{Study Participants}

We enrolled patients who had been discharged from the study ED and agreed to participate in a patient satisfaction survey delivered via their personal phones. We included patients who: 1) recognized physicians that directly involved in their index ED care and 2) were able to rate their trust to physician and rate whether their physicians were showing respect to them. We excluded patients who: 1) refused to participate in this survey; 2) did not know who their physicians were; 3) refused to answer whether they trusted their physicians' care; and 4) refused to answer whether their physicians showed respect to patients. In addition, physicians with less than 10 patient satisfaction surveys were excluded due to lack of statistical significance (Figure 1).

\section{Study Outcomes}

Patient satisfaction surveys were measured as the study outcomes. We used the National Research Corporation (NRC) Picker patient satisfaction survey via telephone to recently discharged ED patients. NRC patient satisfaction survey is one of the most used patient satisfaction survey in the market. ${ }^{15-17}$ NRC picker surveys have several formats and can be delivered either by mail or telephone. All these surveys have been validated externally and used broadly across national EDs. There are 11 questions delivered to patients. In this study, two outcomes from the

2484 Patients Participated in the Survey (October 1, 2018 to September 30, 2019)

287 Patients Not Rated Their Trust or Respect to Healthcare Providers

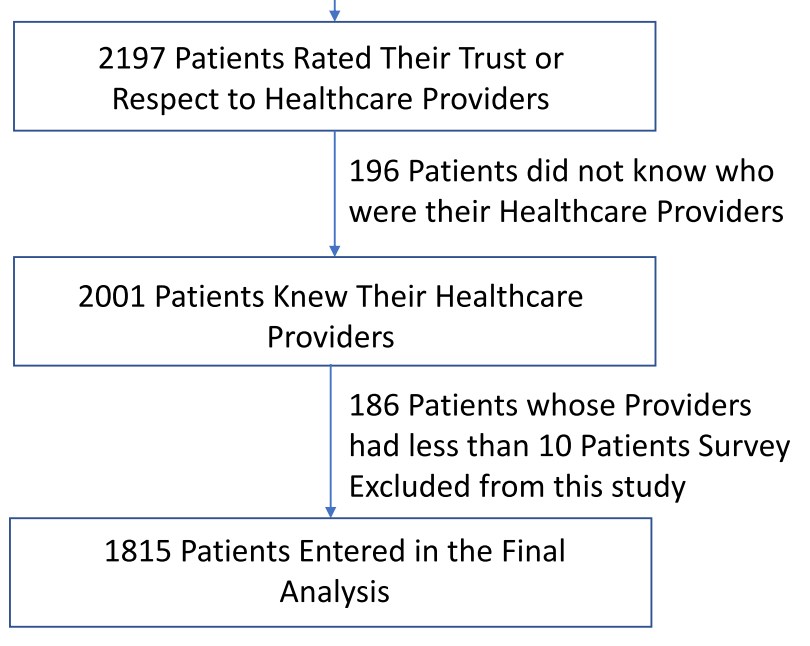

Figure I Study flow diagram. 
survey were measured. One is patient trust physicians' care (hereinafter referred to as "Patient-Trust-Physician," PTP) and the other is physician showing respect to patients (hereafter referred to as "Physician-Show-Respect," PSR). Both questions are answered using a 4-point Likert scale ranging from 1 to 4 . A score of 1 is defined as patients do not trust their physician or a physician does not show any respect to the patient. A score of 4 is defined as patients definitely trust physicians' care and physicians definitely show respect to patients.

\section{Study Variables}

We included both patient and ED physician general demographics including age, gender, race, and ethnicity. The time interval between patient discharge and their completed survey was calculated. Age was divided into three groups: 1) $<40$ years old, 2) between 40 and 54 years old, inclusive (4054 years old), and 3 ) equal to or greater than 55 years old ( $\geq 55$ years old). Race was classified into three groups: 1) White, 2) African American, and 3) Others. "Others" includes Asian, American Indian or Alaska Native, Native Hawaiian or Other Pacific Islanders. Ethnicity was divided into Hispanic and non-Hispanic groups.

\section{Causal Effect Analysis}

We used a counterfactual approach to examine the causal effects of patient-provider demographic concordance on patient perception of physician trust and respect. The counterfactual approach is grounded on the counterfactual theory of causation, which explains the meaning of causal claims in terms of counterfactual conditionals of the form. ${ }^{18,19} \mathrm{We}$ denoted $Y_{i}^{T}$ the outcome (ie, perceived trust and respect by patients) for unit iif the unit receives a treatment $T$. The treatments of particular interest included the patient-physician concordance on four demographic attributes: age, gender, race, and ethnicity. We denoted $Y_{i}^{C}$ the potential outcome for unit $i$ in the control regime $C$ (eg, demographic discordance). Hence, the treatment effect on unit $i$ can be derived by $\tau_{i}=$ $Y_{i}^{T}-Y_{i}^{C}$ We employed genetic matching (GM) to assign patients appropriately to the treatment and control groups. Detailed technical description of the GM algorithm can be found in Sekhon's study. ${ }^{20}$ The absolute standardized mean differences were measured for all covariates before and after the matching to determine the matching performance. ${ }^{20}$ After matching, the joint distributions of patient demographics (eg, age, gender, race, and ethnicity) in the treatment and control groups should have no statistically significant difference.
We then used two-sample $t$-test to determine the causal effects. The $\mathrm{p}$ value $<0.05$ indicates that there is a statistically significant difference between the means of the treatment and control groups, and, therefore, a causal effect exists. Whereas $\mathrm{p}$ value $>0.05$ indicates no causal effect exists based on statistical evidence. All analyses were performed using R package (x64 3.2.5) or STATA 14.2 software (College Station, TX) with p-value $<0.05$ considered statistically significant.

\section{Results}

A total of 1815 patients and 33 ED providers were enrolled in this study. The median time interval from patient discharged from ED to their response to the survey was $67 \mathrm{~h}$ (IQR 43, 104). A detail study flow diagram is shown in Figure 1.

The general demographics were different between patients and physicians. In patients, females were predominant, approximately 40\% were 40-54 years old, and nearly $40 \%$ were Hispanic/Latino patients. Whereas, in physicians, most were male (70\%), White (73\%), and nonHispanic/Latino (94\%) providers (see Table 1).

Figure 2 presents the absolute standardized mean differences for all covariates before and after the GM. According to the plots, the value of absolute standardized

Table I Study Population Demographics

\begin{tabular}{|l|c|c|}
\hline & $\begin{array}{c}\text { Physicians } \\
\text { (n=33) }\end{array}$ & $\begin{array}{c}\text { Patients } \\
\text { (n=18 15) }\end{array}$ \\
\hline Age - n (\%) & & $725(39.9)$ \\
$<40$ years old & $12(36.4)$ & $488(26.9)$ \\
$40-54$ years old & $16(48.5)$ & $595(32.8)$ \\
$\geq 55$ years old & $5(15.2)$ & $7(0.4)$ \\
Unknown & & \\
\hline Gender - n (\%) & $23(69.7)$ & $810(44.6)$ \\
Male & $10(30.3)$ & $1005(55.4)$ \\
Female & & $619(34.1)$ \\
\hline Race - n (\%) & $24(72.7)$ & $528(29.1)$ \\
White & $1(3.0)$ & $662(36.5)$ \\
African American & $8(24.2)$ & $5(0.3)$ \\
Other* & & \\
Unknown or Refused & & $1108(61.1)$ \\
\hline Ethnicity - n (\%) & $2(6.1)$ & $8(0.4)$ \\
Hispanic or Latino & $31(93.9)$ & \\
Not Hispanic/Latino & & \\
Unknown or Refused & & \\
\hline
\end{tabular}

Notes: *Others in Physicians indicate Asian, Others in Patients indicates Nonwhite Hispanic/Latino, Asian, American Indian or Alaska Native, Native Hawaiian or Other Pacific Islanders, etc. 


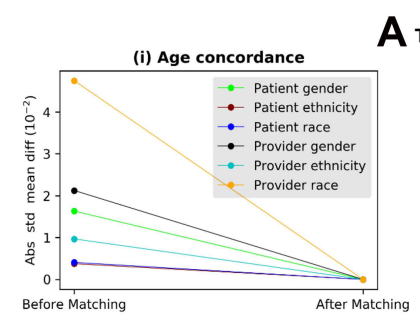

(iii) Ethnicity concordance

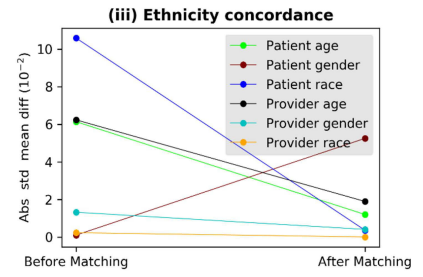

Trust

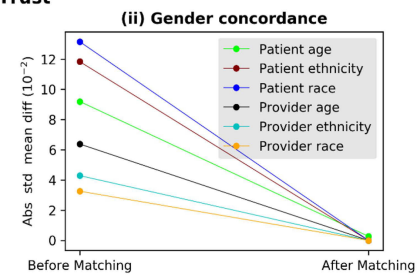

(iv) Race concordance

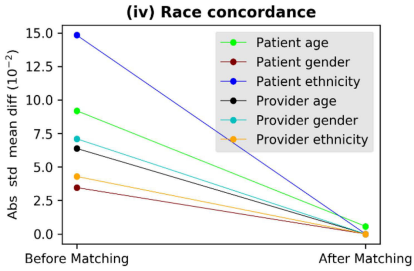

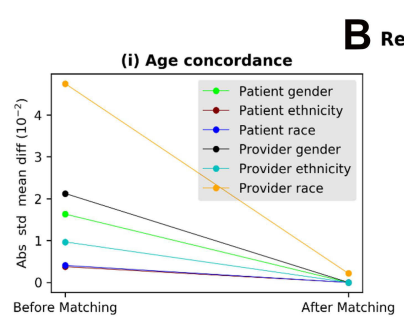

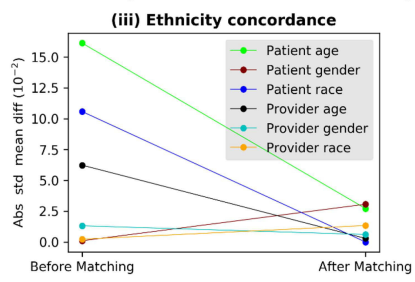

Respect
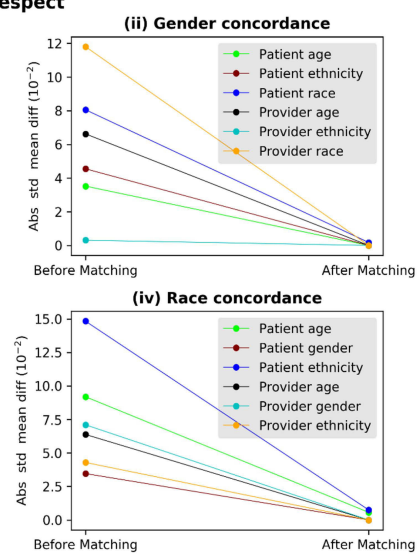

Figure 2 The absolute standardized mean differences for all covariates before and after the genetic matching.

mean differences for all the covariates decreased significantly after the GM, indicating a desirable balance was achieved and the treatment and control groups were prepared for a reliable causal analysis.

Table 2 shows the treatment effects of gender, age, race, and ethnicity concordance on patient perception of physician trust and respect. In all cases, the treatment effect is negative. However, only the treatment effect of age concordance on physician trust is statistically significant $(p=0.036)$. This indicates that patient-physician age concordance may cause a negative effect on patient's trust to their physicians. The rest of the estimated effects are not statistically significant.

\section{Discussion}

Physicians showing respect to patients and gaining patient trust during healthcare process are critical to establish patient-physician rapport, which subsequently affects patient health-care outcomes. ${ }^{21-23}$ However, patient perceptions of physician trust and respect are quite subjective and can be affected by multiple factors. Each potential factor can have interactions, thus making routine association analyses (eg, correlation, regression, etc.) less reliable. ${ }^{24}$ Therefore, we performed a causal effect analysis in this study. We found that other than patient-physician age concordance could cause a negative effect on patient perception of physician trust, patient-physician demographics, in general, minimally affected patient perceptions of physician trust and respect. To the best of our knowledge, such direct causative effect analyses, specifically on patient perceptions of physician trust and respect in related to patient-physician demographics, has not been reported before. Our findings add extra evidence to the literature on demographics affecting patient-physician rapport. Such findings might help future improvement in physician communication training during emergency care.

Table 2 Treatment Effects on Patient Perceptions of Patient-Trust-Physician and Physician-Show-Respect

\begin{tabular}{|l|l|l|l|}
\hline Treatments & Estimated Effect & T-Stat & p-value \\
\hline \multicolumn{1}{|c|}{ Patient perceptions of patient-trust-physician } \\
\hline Gender concordance & -0.024 & -0.484 & 0.629 \\
Age concordance & -0.119 & -2.097 & 0.036 \\
Race concordance & -0.043 & -0.729 & 0.466 \\
Ethnicity concordance & -0.181 & -1.595 & 0.111 \\
\hline & Patient perceptions of physician-show-respect & 0.672 \\
\hline Gender concordance & -0.022 & -0.423 & 0.071 \\
Age concordance & -0.102 & -1.805 & 0.378 \\
Race concordance & -0.051 & -0.882 & 0.129 \\
Ethnicity concordance & -0.200 & -1.516 & \\
\hline
\end{tabular}


The study's strength is using causal effect analysis in an observational data setting. Such counterfactual analysis to causation for a general problem was reported before, but not widely used due to strict model requirement. ${ }^{25}$ Causal models are constructed separately to identify the causal relationship between each outcome and each treatment, it is ideal to measure cause effects on random samples. However, in an observational data setting, the baseline variables are almost never balanced across the treatment and control groups because the two groups are not ordinarily drawn from the same population. Therefore, matching a procedure used to derive a balance of the baseline variables across treatment and control groups - must be conducted prior to the estimation of causal effects. This study employs genetic matching (GM) - a well-known matching method - to select patient-physician encounters from observational data and assign them appropriately to treatment and control groups. ${ }^{20,26}$ Using such matched samples could thus determine the direct causal effects accurately.

Previous studies favored patient-physician demographic concordance in certain specialties (eg, gynecology and family practice) ${ }^{27,28}$ It can be inferred that patientphysician demographic concordance may affect communication patterns and perception of quality of care that can further affect patient-physician relationship and health outcomes. However, such perceptions may not be duplicated in an emergent care setting. In an emergent care setting, patient-physician rapport is temporary and can be affected significantly by limited time and communication. At present, the influence of ED physician demographics affecting patient-centered care is not as well studied. A recent report of an ED study showed patientadvocate demographic concordance has no effect on the promotion of ED patients' HIV screening, though this is not strictly patient-provider related. ${ }^{29}$ On the other hand, we are unclear of patient-physician age concordance having negative effect on patient perception of physician trust in our study. A previous study reported that patient age acted as an independent factor in predicting the trust to their physician among hospitalized trauma patients. ${ }^{7}$ However, another study showed that patients' views regarding age concordance were varied and unrelated to gender or racial/ethnic group. ${ }^{28}$ Our future study will be particularly focused on the patient-physician age concordance in relation to ED patient-centered care.

\section{Limitation}

Our study has its limitations. This is a retrospective observational study with secondary data analysis. Given the nature of this study design, patient selection bias, missing data, and inaccurate information inevitably exist. We excluded over $20 \%$ of the initial data due to our exclusion criteria without data imputation, and such exclusion might generate potential bias for result interpretation. Although this study enrolled significant patient samples, our physician sample size is limited, which could potentially affect patient physician generic matching. Additionally, risks affecting PTP/PSR are multifactorial, in this study, we only addressed the causal effect in terms of the patient-physician demographic features. The causal associations between PTP/PSR and other risks (eg, patients' education levels, patient health-care conditions, or ED length of stay, etc.) were not analyzed in this study. Moreover, while this study was focused on PTP/PSR, overall patient satisfaction was not specifically investigated, which might be hard to compare with other studies. Therefore, to accurately determine the role of patient-physician demographic concordance in relation to patientcentered care, a larger multi-center prospective study is warranted for further validation.

\section{Conclusion}

When a patient's near real-time satisfaction was performed in an emergent care setting, patient-physician age concordance may cause a negative effect on patient's trust to their physicians. Otherwise, patient-physician demographic concordance has minimal causal effect on patient perceptions of physician trust and respect.

\section{Abbreviations}

ED, Emergency Department; PTP, Patient-trust-physician; PSR, Physician-show-respect; NRC, National research corporation; GM, genetic matching.

\section{Data Sharing Statement}

The data from this study are not available due to patient confidentiality reasons but are available upon request to the corresponding author.

\section{Ethics Approval and Consent to Participate}

This study is waived for approval of documentation of informed consent by University of North Texas 
Institutional Review Board due to a quality improvement project with retrospective data analysis.

\section{Author Contributions}

All authors made a significant contribution to the work reported, whether that is in the conception, study design, execution, acquisition of data, analysis, and interpretation, or in all these areas; took part in drafting, revising, or critically reviewing the article; gave final approval of the version to be published; have agreed on the journal to which the article has been submitted; and agree to be accountable for all aspects of the work.

\section{Funding}

There is no funding to report.

\section{Disclosure}

The authors report no conflicts of interest in this work.

\section{References}

1. Scott A, Smith RD. Keeping the customer satisfied: issues in the interpretation and use of patient satisfaction surveys. Int $J$ Qual Health Care. 1994;6(4):353-359. doi:10.1093/intqhe/6.4.353

2. Ford RC, Bach SA, Fottler MD. Methods of measuring patient satisfaction in health care organizations. Health Care Manage Rev. 1997;22:74-89. doi:10.1097/00004010-199704000-00009

3. Brown T, Shetty A, Zhao DF, Harvey N, Yu T, Murphy M. Association between pain control and patient satisfaction outcomes in the emergency department setting. Emerg Med Australas. 2018;30 (4):523-529. doi:10.1111/1742-6723.12945

4. Abolfotouh MA, Al-Assiri MH, Alshahrani RT, Almutairi ZM, Hijazi RA, Alaskar AS. Predictors of patient satisfaction in an emergency care centre in central Saudi Arabia: a prospective study. Emerg Med J. 2017;34(1):27-33. doi:10.1136/emermed-2015-204954

5. McFarland DC, Ornstein KA, Holcombe RF. Demographic factors and hospital size predict patient satisfaction variance-implications for hospital value-based purchasing. J Hosp Med. 2015;10:503-509. doi:10.1002/jhm.2371

6. McFarland DC, Shen MJ, Holcombe RF. Predictors of patient satisfaction with inpatient hospital pain management across the United States: a national study. J Hosp Med. 2016;11(7):498-501. doi: $10.1002 / \mathrm{jhm} .2576$

7. Ommen O, Janssen C, Neugebauer E, Pfaff H. Determinants of severely injured patients' trust in their hospital physicians. Chirurg. 2007;78(1):52-61. doi:10.1007/s00104-006-1229-9

8. Pinder RJ, Ferguson J, Moller H. Minority ethnicity patient satisfaction and experience: results of the national cancer patient experience survey in England. BMJ Open. 2016;6(6):e011938. doi:10.1136/ bmjopen-2016-011938

9. Morgan MW, Salzman JG, LeFevere RC, Thomas AJ, Isenberger KM. Demographic, operational, and healthcare utilization factors associated with emergency department patient satisfaction. West J Emerg Med. 2015;16(4):516-526. doi:10.5811/ westjem.2015.4.25074

10. Sohler NL, Fitzpatrick LK, Lindsay RG, Anastos K, Cunningham CO. Does patient-provider racial/ethnic concordance influence ratings of trust in people with HIV infection? AIDS Behav. 2007;11:884-896. doi:10.1007/s10461-007-9212-0
11. Street RL Jr, O'Malley KJ, Cooper LA, Haidet P. Understanding concordance in patient-physician relationships: personal and ethnic dimensions of shared identity. Ann Fam Med. 2008;6(3):198-205. doi: $10.1370 / \mathrm{afm} .821$

12. Kumar D, Schlundt DG, Wallston KA. Patient-physician race concordance and its relationship to perceived health outcomes. Ethn Dis. 2009; 19:345-351.

13. Crawford D, Paranji S, Chandra S, Wright S, Kisuule F. The effect of racial and gender concordance between physicians and patients on the assessment of hospitalist performance: a pilot study. BMC Health Serv Res. 2019;19(1):247. doi:10.1186/s12913-019-4090-5

14. Shen MJ, Peterson EB, Costas-Muniz R, et al. The effects of race and racial concordance on patient-physician communication: a systematic review of the literature. $J$ Racial Ethn Health Disparities. 2018;5:117-140. doi:10.1007/s40615-017-0350-4

15. Haviland MG, Pincus HA, Morales LS. Depression and satisfaction with health coverage and medical care in the 1998 NRC healthcare market guide survey. Adm Policy Ment Health. 2003;30:511-522. doi:10.1023/A:1025079101897

16. Haviland MG, Morales LS, Dial TH, Pincus HA. Race/ethnicity, socioeconomic status, and satisfaction with health care. Am J Med Qual. 2005;20:195-203. doi:10.1177/1062860605275754

17. Co JP, Sternberg SB, Homer CJ. Measuring patient and family experiences of health care for children. Acad Pediatr. 2011;11:S59S67. doi:10.1016/j.acap.2011.01.009

18. Kim J. Causes and counterfactuals. J Philos. 1973;70:570-572. doi: $10.2307 / 2025312$

19. Kaiserman A. Causes and counterfactuals. Australas $J$ Philos. 2017;95:17-28. doi:10.1080/00048402.2016.1157695

20. Sekhon JS. Multivariate and propensity score matching software with automated balance optimization: the matching package for R. J Stat Softw. 2011;42:1-52. doi:10.18637/jss.v042.i07

21. Balkrishnan R, Dugan E, Camacho FT, Hall MA. Trust and satisfaction with physicians, insurers, and the medical profession. Med Care. 2003;41:1058-1064. doi:10.1097/01.MLR.0000083743.15238.9F

22. Jneid S, Jabbour H, Hajj A, et al. Quality of life and its association with treatment satisfaction, adherence to medication, and trust in physician among patients with hypertension: a Cross-Sectional Designed Study. J Cardiovasc Pharmacol Ther. 2018;23:532-542. doi: $10.1177 / 1074248418784292$

23. Sokol-Hessner L, Folcarelli PH, Annas CL, et al. A road map for advancing the practice of respect in health care: the Results of an Interdisciplinary Modified Delphi Consensus Study. Jt Comm J Qual Patient Saf. 2018;44:463-476.

24. Saccenti E, Hendriks MHWB, Smilde AK. Corruption of the Pearson correlation coefficient by measurement error and its estimation, bias, and correction under different error models. Sci Rep. 2020;10:438. doi:10.1038/s41598-019-57247-4

25. Hofler M. Causal inference based on counterfactuals. BMC Med Res Methodol. 2005;5:28. doi:10.1186/1471-2288-5-28

26. Diamond A, Sekhon JS. Genetic matching for estimating causal effects: a general multivariate matching method for achieving balance in observational studies. Rev Econ Stat. 2013;95:932-945. doi:10.1162/REST_a_00318

27. Rogo-Gupta LJ, Haunschild C, Altamirano J, Maldonado YA, Fassiotto M. Physician gender is associated with press Ganey patient satisfaction scores in outpatient gynecology. Womens Health Issues. 2018;28(3):281-285. doi:10.1016/j.whi.2018.01.001

28. Garcia JA, Paterniti DA, Romano PS, Kravitz RL. Patient preferences for physician characteristics in university-based primary care clinics. Ethn Dis. 2003;13:259-267.

29. Aronson ID, Cleland CM, Rajan S, Marsch LA, Bania TC. Computer-based substance use reporting and acceptance of HIV testing among emergency department patients. AIDS Behav. 2020;24(2):475-483. doi:10.1007/s10461-019-02517-5 
Open Access Emergency Medicine

Dovepress

\section{Publish your work in this journal}

The Open Access Emergency Medicine is an international, peerreviewed, open access journal publishing original research, reports, editorials, reviews and commentaries on all aspects of emergency medicine. The manuscript management system is completely online

and includes a very quick and fair peer-review system, which is all easy to use. Visit http://www.dovepress.com/testimonials.php to read real quotes from published authors.

Submit your manuscript here: https://www.dovepress.com/open-access-emergency-medicine-journal 